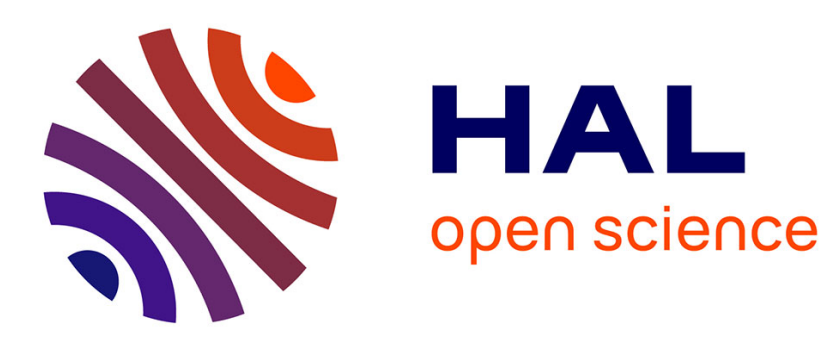

\title{
Travail et pauvreté en Russie: évaluations objectives et perceptions subjectives
}

Ekaterina Kalugina, Boris Najman

\section{To cite this version:}

Ekaterina Kalugina, Boris Najman. Travail et pauvreté en Russie: évaluations objectives et perceptions subjectives. Economie et Statistique / Economics and Statistics, 2004, 367 (février 2004), pp.83-100. hal-00266727

\section{HAL Id: hal-00266727 https://hal.science/hal-00266727}

Submitted on 25 Mar 2008

HAL is a multi-disciplinary open access archive for the deposit and dissemination of scientific research documents, whether they are published or not. The documents may come from teaching and research institutions in France or abroad, or from public or private research centers.
L'archive ouverte pluridisciplinaire HAL, est destinée au dépôt et à la diffusion de documents scientifiques de niveau recherche, publiés ou non, émanant des établissements d'enseignement et de recherche français ou étrangers, des laboratoires publics ou privés. 


\title{
Travail et pauvreté en Russie : évaluations objectives et perceptions subjectives
}

\author{
Ekaterina Kalugina et Boris Najman*
}

La relation entre la pauvreté et la participation au marché du travail en Russie est analysée à l'aide de deux mesures de la pauvreté : une mesure monétaire en termes de seuil absolu et une mesure subjective selon l'autoévaluation du niveau de pauvreté. Les données individuelles de panel utilisées sont tirées de l'enquête RLMS (Russia Longitudinal Monitoring Survey) entre 1994 et 2000.

Il est nécessaire de prendre en compte les statuts (formel, informel ou pluri-activité) sur le marché du travail russe afin de comprendre quels sont les risques de pauvreté auxquels font face les personnes ayant un emploi. Observer seulement la participation au marché du travail (emploi/non-emploi) ne suffit pas.

Un résultat essentiel est que le fait d'avoir un seul emploi déclaré (secteur formel) augmente la probabilité d'être et de se sentir pauvre relativement au fait de travailler dans le secteur informel. À l'opposé, les personnes ayant plusieurs activités ont la probabilité la plus faible d'être ou de se sentir pauvre. L'emploi salarié (formel) correspond à la situation matérielle la plus précaire aujourd'hui en Russie.

* Ekaterina Kalugina appartient au Team-Université de Paris 1 et au HCE de Moscou. Boris Najman appartient au RosesUniversité de Paris 1 et au Gratice-Université de Paris 12.

Les noms et dates entre parenthèses renvoient à la bibliographie en fin d'article. 
A u cours des dix dernières années, les pays en transition, et plus particulièrement ceux de la Communauté des États Indépendants (CEI) (1), ont connu une évolution très contrastée de leur PIB. En Russie, le niveau du PIB réel en 1999 a atteint 55 si l'on prend 100 comme base pour 1989 (2). Cette chute a été précédée ou accompagnée d'une très forte inflation (Banque Mondiale, 1996). Celle-ci a dépassé $1000 \%$ en moyenne dans les pays de la CEI durant trois années consécutives (1992-1994) (3), et a directement affecté la baisse des salaires réels, qui atteignait en Russie 43,6\% entre 1993 et 1999 (BERD, 2001). Elle a également contribué à augmenter la part de la population située en dessous du seuil de pauvreté, qui représentait $30 \%$ de la population russe totale en 1998. À partir de 1999, les indicateurs macroéconomiques sont redevenus positifs (4). Mais l'effet de la croissance sur la réduction des inégalités et de la pauvreté est jusqu'à présent resté très limité. Malgré une croissance du PIB en termes réels de plus de $14 \%$ depuis 1998 , la part de la population en dessous du seuil de pauvreté reste proche de $30 \%$. Les revenus des $10 \%$ les plus riches excèdent ceux des $10 \%$ les plus pauvres de presque 14 fois. Le coefficient de Gini (5) et le niveau de pauvreté sont très élevés (6). À l'heure actuelle, le système de protection sociale russe ne répond pas aux besoins des personnes les plus vulnérables, malgré les différentes tentatives des autorités pour le réformer. Le système tel qu'il existe n'est pas efficace, et se caractérise par des prestations très faibles et universelles (cf. tableau $\mathrm{C}$ en annexe 1 ). Il n'intègre presque aucun transfert individualisé (7).

La littérature économique récente (Lollivier et Verger, 1997) sur la pauvreté insiste sur le fait qu'il s'agit d'un phénomène multidimensionnel et que la situation matérielle ne correspond pas systématiquement à l'évaluation que font les agents eux-mêmes de leur pauvreté relative (8). Les données d'enquête sur la Russie (Russia Longitudinal Monitoring Survey, RLMS, vagues 5 à 9, 1994-2000) (9) permettent d'évaluer les déterminants de la perception subjective et ceux de la pauvreté objective. On se propose donc d'étudier les déterminants des deux mesures de la pauvreté en se concentrant sur les différents statuts du marché du travail russe. Dans un contexte de forte de réduction des revenus du secteur formel et de crise du salariat, il est en effet nécessaire de prendre en compte la diversité des activités possibles (c'est-à-dire le secteur informel et la pluri-activité) car les activités complémentaires, informelles ou non, permettent d'améliorer la situation matérielle des indivi- dus, et la perception qu'ils ont de leur richesse. Un résultat significatif porte sur la relation entre participation au secteur informel et pauvreté. Le fait de travailler dans le secteur informel, comme activité unique et exclusive, diminue la probabilité d'être ou de se sentir pauvre, par rapport au statut d'emploi formel.

\section{Comment mesurer la pauvreté : être ou se sentir pauvre?}

L'idée principale de l'approche subjective est que la pauvreté ne se base plus sur le minimum vital (approche absolue) ou l'état des conditions de vie (approche relative), mais sur la perception qu'ont les ménages de l'aisance dans laquelle ils vivent. Les individus sont supposés connaître de façon assez précise leur situation matérielle et, de plus, être capables de l'évaluer de manière relative.

Une des mesures subjectives de la pauvreté couramment utilisée repose sur une question rela-

1. Union libre d'États indépendants regroupant 12 des 15 anciennes républiques soviétiques. Elle a été créée en décembre 1991. Républiques membres : Arménie, Azerbaïdjan (depuis septembre 1993), Biélorussie, Géorgie (depuis décembre 1993), Kazakhstan, Kirghizistan, Moldavie, Ouzbékistan, Russie (Fédération de), Tadjikistan, Turkménistan, Ukraine.

2. Sur l'ensemble des pays de la CEl, l'indice est de 53 pour 1998 ; pour les pays d'Europe centrale et orientale, il est de 95 pour la même année. (Banque européenne de reconstruction et de développement, BERD, 2001).

3. Pour la CEl, l'inflation calculée à partir de l'indice des prix à la consommation mesurée en fin d'année (BERD, 2001) a été en moyenne respectivement pour les trois années de $1672 \%$, $4585 \%$ et $1391 \%$.

4. Le PIB augmente en 1999 et 2000 et le chômage baisse en 2000 (cf. tableau A en annexe 1). En 2000, le taux de chômage selon la définition du BIT est de $8,4 \%$ de la population en âge de travailler.

5. Le coefficient de Gini est un chiffre entre zéro et un qui mesure le degré d'inégalité dans la distribution des revenus dans un pays donné. Le coefficient enregistrerait zéro inégalité $(0,0=$ inégalité minimale) pour une société dans laquelle chaque membre recevrait exactement le même revenu et enregistrerait un coefficient de un $(1,0=$ inégalité maximale) si un membre recevait tous les revenus et les autres rien.

6. Cf. tableau B en annexe 1, Comité pour les Statistiques en Russie (Goskomstat, 2001).

7. De plus, un grand nombre de prestations sociales (par exemple, les frais d'hospitalisation) qui dans les textes sont prises en charge par l'État sont en réalité payées par les usagers.

8. Dans la littérature économique, on considère généralement trois méthodes distinctes de mesure de la pauvreté (fixation du seuil de pauvreté) : absolue, relative et subjective. La notion de pauvreté absolue se réfère à la satisfaction des besoins essentiels: nourriture, logement, vêtements, soins médicaux. Les médecins déterminent la consommation minimale de calories par individu pour assurer un état de santé satisfaisant. La notion de pauvreté relative se réfère à la distribution des revenus et correspond à un indicateur d'inégalités. Par exemple, on considère comme pauvre tout individu qui dispose d'un revenu inférieur à la moitié du revenu médian (ou du revenu moyen). La troisième notion de pauvreté est celle de pauvreté subjective qui repose sur des enquêtes d'opinion.

9. Toute l'information sur les données de RLMS peut être trouvée sur la page web du projet où on peut obtenir les données gratuitement : http ://www.cpc.unc.edu/rlms. 
tive à l'échelle du bien-être individuel : il est demandé à chaque personne de se situer sur une échelle de plusieurs paliers allant de manière croissante de «pauvre » à « riche ». C'est ce type de question qui est présente dans l'enquête RLMS et que l'on utilise dans cet article: "Imaginez, une échelle à neuf paliers où sur le palier le plus bas se trouvent les gens les plus pauvres et sur le palier le plus haut les gens les plus riches. Sur quel palier vous placeriezvous?».

L'estimation subjective, comme toutes les mesures de la pauvreté, présente des inconvénients. Les réponses peuvent être influencées par des facteurs divers, comme les attentes ou les habitudes de chacun. Elles peuvent également se référer à un niveau jugé moyen au niveau du lieu d'habitation, ou dans un sousgroupe de référence (catégorie socioprofessionnelle, famille, etc.), ou encore par rapport à un niveau jugé normal pour d'autres raisons (comme le niveau des minima sociaux).

L'échelle de neuf paliers subjectifs a été transformée en variable dichotomique. Initialement à neuf modalités, cette variable prend la valeur 1 lorsque l'individu interrogé se situe dans les catégories 1 ou 2, donc pauvre; sinon, elle est égale à 0 (10).

Une personne est considérée comme objectivement pauvre si les revenus nominaux totaux du ménage sont inférieurs au seuil de pauvreté régional, lui-même exprimé en termes nominaux. La base $R L M S$ permet d'estimer le revenu nominal total du ménage, qui inclut les salaires, les prestations sociales, les transferts privés ainsi que l'estimation monétaire de la production agricole (11). La ligne de pauvreté régionale est construite dans le cadre du projet RLMS. Elle s'inspire des travaux de Popkin et al. (1992 et 1995) qui se fondent sur le calcul du nombre de calories nécessaire à la survie d'un individu selon sa catégorie démographique. Cette méthode dite absolue détermine un panier de biens de première nécessité, élaboré séparément par tranche d'âge, pour les enfants de 0 à 6 ans, les enfants et adolescents de 7 à 17 ans, les adultes hommes et femmes, les hommes retraités de 60 ans et plus, les femmes retraitées de 55 ans et plus. Ensuite, des prix spécifiques régionaux sont utilisés pour calculer les coûts du panier de consommation. Chaque catégorie de la population, en fonction de son âge, de son sexe et de sa région d'appartenance, a donc sa propre ligne de pauvreté qui est utilisée pour construire la ligne de pauvreté du ménage (12).
La variable que l'on construit pour mesurer la pauvreté objective comporte deux modalités. Elle est égale à 1 , si le revenu nominal total du ménage est inférieur au seuil de pauvreté régionale en termes de revenu nominal corrigé de la structure du ménage, sinon elle est égale à zéro.

Les proportions des personnes vivant en dessous du seuil de pauvreté absolu ou se sentant pauvres sont du même ordre (près de $25 \%$ en 2000 , cf. tableau 1), cependant les personnes situées en dessous du seuil de pauvreté ne correspondent pas aux personnes se considérant comme pauvres. En 2000, parmi les 2045 personnes qui se sont placées comme pauvres selon l'échelle subjective, 1264 (plus de $60 \%$ ) ne sont pas pauvres selon l'estimation objective de la pauvreté et parmi 2459 personnes étant pauvres selon la mesure objective, 1678 (plus de $68 \%$ ) considèrent qu'elles ne sont pas pauvres (cf. tableau 2). Les pauvretés objective et subjective ne concernent donc pas les mêmes personnes.

Le non-recouvrement des mesures est liée aux divergences existantes entre les deux méthodes. Il souligne que les mesures monétaires ne prennent pas en compte l'ensemble des caractéristiques de la pauvreté dont l'emploi et le statut sur le marché du travail font partie. On s'est donc demandé si la participation au marché du travail

\footnotetext{
10. À partir du palier 6, le nombre d'observations est très réduit. Par exemple, en 2000 seules 34 personnes se sont placées sur le palier 7, 5 le palier 8,5 encore sur le palier 9 et cela parmi 8481 individus ayant répondu à la question portant sur l'échelle de pauvreté subjective.

11. Il s'agit d'agriculture domestique sur les lopins individuels et de la consommation du ménage. Elle est évaluée en fonction des prix du marché. Ces prix ont été collectés par les observateurs de RLMS dans les magasins et marchés locaux.

12. La ligne de pauvreté du ménage est une somme des lignes de pauvreté individuelles à l'intérieur du ménage corrigée en fonction de la taille du ménage. Les coefficients varient donc en fonction de la taille du ménage: 0,90 pour un ménage de 2 personnes; 0,82 si la taille du ménage est égale à $3 ; 0,76$ pour 4 personnes; 0,72 pour $5 ; 0,69$ pour 6 et 0,68 si la taille $d u$ ménage est supérieure à 6 personnes.
}

\section{Tableau 1 \\ Comparaison des estimations objectives et subjectives de la pauvreté}

\begin{tabular}{|c|c|c|}
\hline Année & $\begin{array}{c}\text { Individus en dessous du } \\
\text { seuil de pauvreté objective }\end{array}$ & $\begin{array}{c}\text { Individus se situant sur les } \\
\text { deux premiers paliers }\end{array}$ \\
\hline 1994 & 15,2 & 28,1 \\
1995 & 28,7 & 30,6 \\
1996 & 34,7 & 30,2 \\
1998 & 38,8 & 35,5 \\
2000 & 27,9 & 24,1 \\
\hline
\end{tabular}

Source : RLMS, vagues 5-9 (1994-2000). 
avait le même impact sur la probabilité d'être et sur la probabilité de se sentir pauvre. L'emploi (surtout, quel type d'emploi) permet-il d'être au-dessus du seuil de pauvreté, d'atteindre un niveau de bien-être supérieur? Observe-t-on une différence entre pauvreté objective et perception subjective selon le statut sur le marché du travail (formel, informel, pluri-activité) ?

\section{La prise en compte de la pluri-activité et des activités informelles}

Les statuts sur le marché du travail que l'on a élaborés prennent en compte les particularités du marché du travail russe, en particulier la pluri-activité et les activités informelles (cf. tableau 3). On définit de manière exclusive les différentes activités sur le marché du travail comme suit (13) :

- avoir un seul emploi salarié (emploi formel) : les personnes qui ont un emploi principal dans une organisation, une entreprise, une administration, c'est-à-dire une structure légale et qui les déclare, ou qui sont entrepreneurs. Cet emploi est unique ;

- activité informelle : les personnes ayant de petites activités individuelles et celles ayant un emploi non déclaré ou situé en dehors d'une structure institutionnelle légale. Cette catégorie n'autorise pas non plus le cumul d'activité ;

- la pluri-activité regroupe les personnes ayant deux activités au moins, qui peuvent cumuler, en plus de leur emploi principal, une activité secondaire (14) ou/et activité informelle. Cette catégorie a été particulièrement étudiée dans les pays en transition (BERD, 2000).

Beaucoup de personnes sont obligées de travailler dans plusieurs emplois et/ou dans le secteur informel (Najman, 2000) en raison du très faible niveau de salaire dans certains secteurs de l'économie, ainsi que des arriérés de salaire. Selon les estimations du Bureau international du travail (BIT) (15), 11,6\% de la population active en Russie participe au secteur informel. L'économie informelle n'est pas un phénomène nouveau pour la Russie. L'économie souterraine existait déjà à l'époque soviétique (16) (Grossman, 1983), mais l'augmentation considérable de celle-ci est liée au processus de transition de la Russie vers l'économie de marché. Ce type d'activité est un moyen de se soustraire aux impôts et d'avoir un revenu d'appoint lorsque le salaire est trop faible. La pratique de payer les employés au noir est répandue, même dans les entreprises légales (Kaliberda et Kaufmann, 1996). D’après le BIT, un cinquième des employés travaillant dans les entreprises officielles perçoit plus que le salaire déclaré dans son contrat.

Malheureusement, on n'a pas de possibilité de mesurer ce que l'on peut appeler une rémunération informelle dans les entreprises du secteur formel. L'enquête $R L M S$ ne fournit pas d'information sur les modes de paiements des salariés dans l'entreprise. La catégorie d'activité informelle dans l'enquête RLMS concerne donc surtout les petites activités non déclarées par les enquêtés au cours des 30 derniers jours (17). On

\footnotetext{
13. Les auteurs tiennent à remercier Jérémie Gignoux pour son aide dans la définition de ces statuts.

14. Les emplois principaux ou secondaires correspondent aux définitions utilisées dans l'enquête RLMS.

15. Cf.Tchetvernina, Moscovskaya, Soboleva et Stepantchikova (2001).

16. Les enquêtes budget des ménages réalisées de 1969 à 1990 révèlent un secteur informel, qui au total augmente de 6,8 \% le niveau du PIB soviétique de l'époque (Kim, 2003).

17. La question présente dans le questionnaire est la suivante "Vous êtes-vous engagé dans une forme de travail (additionnel) pour laquelle vous avez été payé ? ". II s'agit principalement de petites activités de service. Des exemples sont donnés : "Peutêtre avez-vous fait du repassage, conduit quelqu'un, aidé quelqu'un dans la réparation d'une voiture ou d'un appartement, acheté et livré de la nourriture ou des biens dans un marché ou dans la rue, ou exercé une autre activité pour laquelle vous avez été payé ?".
}

Tableau 2

Pauvreté objective et subjective en $\mathbf{2 0 0 0}$

\begin{tabular}{|l|c|c|c|}
\hline \multirow{2}{*}{ Pauvreté subjective } & \multicolumn{3}{|c|}{ Pauvreté objective } \\
\cline { 2 - 4 } & Non pauvre & Pauvre & Total \\
\hline Non pauvre & 4758 & 1678 & 6436 \\
& $(73,93)$ & $(26,07)$ & $(100,00)$ \\
\hline Pauvre & 1264 & 781 & 2045 \\
& $(61,81)$ & $(38,19)$ & $(100,00)$ \\
\hline Total & 6022 & 2459 & 8481 \\
& $(71,01)$ & $(28,99)$ & $(100,00)$ \\
\hline
\end{tabular}

Lecture : les pourcentages sont indiqués en italique et entre parenthèses.

Source : RLMS, vague 9 (2000). 
a aussi englobé dans cette catégorie les personnes qui participent à ces petites activités mais qui peuvent se déclarer par ailleurs comme chômeurs ou comme inactifs, ainsi que ceux qui déclarent une activité principale mais l'exercent en dehors d'une entreprise ou d'une organisation et ne se déclarent pas comme entrepreneurs.

Le statut des pluri-actifs correspond aux individus possédant déjà une première activité principale et une ou plusieurs activités supplémentaires. Cette activité secondaire peut être soit formelle, soit informelle. Dans les deux cas, la personne est considérée comme pluri-active. De

\section{Tableau 3}

Répartition des emplois selon le secteur institutionnel

En \% des personnes employées

\begin{tabular}{|c|c|c|c|}
\hline & $\begin{array}{c}\text { Emploi } \\
\text { formel }\end{array}$ & $\begin{array}{c}\text { Activités } \\
\text { informelles }\end{array}$ & $\begin{array}{c}\text { Pluri- } \\
\text { activité }\end{array}$ \\
\hline 1994 & 82,65 & 6,95 & 10,40 \\
1995 & 82,77 & 8,52 & 8,71 \\
1996 & 82,95 & 8,30 & 8,75 \\
1998 & 79,67 & 11,86 & 8,47 \\
2000 & 76,75 & 14,47 & 8,78 \\
\hline
\end{tabular}

Source: RLMS, vagues d'enquête 5-9.
1994 à 2000, une part stable d'individus (environ $9 \%$ des personnes ayant un emploi, cf. tableau 3 ) est engagée dans plusieurs activités à la fois.

En Russie sur cette période, les individus possédant plusieurs emplois ou travaillant dans le secteur informel représentent environ 20 à $25 \%$ des personnes employées. Cette part a tendance à croître sur l'ensemble de la période, mais au bénéfice des activités informelles (cf. tableau 3). Enfin, la personne est considérée comme possédant un emploi formel si elle déclare avoir une seule activité principale exercée soit dans une organisation/entreprise soit comme indépendant.

On observe tout d'abord que les salaires horaires des activités informelles sont très supérieurs (plus de deux fois) à ceux du secteur formel, surtout pour les pluri-actifs (trois fois plus élevés) (cf. tableau 4). Les rémunérations informelles représentent plus de $40 \%$ du revenu total du ménage pour les personnes qui participent au secteur informel de manière exclusive, plus de $25 \%$ du revenu total du ménage pour les individus pluri-actifs. Ensuite, la participation au secteur informel est limitée en termes de volume horaire : un individu est bien mieux rémunéré pour une heure travaillée dans le secteur informel

Tableau 4

Temps de travail, taux de rémunération des actifs formels et informels et revenus du ménage

\begin{tabular}{|c|c|c|c|c|c|c|}
\hline & \multicolumn{2}{|c|}{ Pluri-actifs } & \multicolumn{2}{|c|}{$\begin{array}{l}\text { Monoactifs } \\
\text { (formels) }\end{array}$} & \multicolumn{2}{|c|}{$\begin{array}{l}\text { Actifs occasionnels } \\
\quad \text { (informels) }\end{array}$} \\
\hline & Femmes & Hommes & Femmes & Hommes & Femmes & Hommes \\
\hline \multicolumn{7}{|l|}{ Activité formelle } \\
\hline Temps de travail & 144,2 & 168,0 & 154,5 & 176,8 & & \\
\hline Temps de travail nul (en \%) & 0,12 & 0,09 & 0,12 & 0,06 & & \\
\hline Salaire ou revenu horaire & 11,6 & 15,4 & 12,1 & 15,5 & & \\
\hline Salaire nul (en \%) & 17,0 & 0,22 & 0,21 & 0,24 & & \\
\hline \multicolumn{7}{|l|}{ Activité informelle } \\
\hline Temps de travail & 51,8 & 46,2 & & & 111,4 & 119,7 \\
\hline Temps de travail nul (en \%) & 3,0 & 3,0 & & & 3,0 & 2,0 \\
\hline Revenu horaire & 33,5 & 63,5 & & & 22,2 & 32,5 \\
\hline Salaire nul (en \%) & 18,0 & 11,0 & & & 17,0 & 16,0 \\
\hline Revenu réel total du ménage & 5580 & 7035 & 4935 & 5145 & 5290 & 5440 \\
\hline $\begin{array}{l}\text { dont : } \\
\text { revenus formels (en \%) }\end{array}$ & 0,47 & 0,50 & 0,60 & 0,62 & 0,22 & 0,18 \\
\hline revenus informels (en \%) & 0,25 & 0,26 & 0,05 & 0,03 & 0,43 & 0,40 \\
\hline
\end{tabular}

Lecture : temps de travail (heures mensuelles) et taux de salaire (salaire horaire en roubles de décembre 2000) dans un emploi formel et dans une activité informelle pour les pluri-actifs, les actifs formels uniques et les actifs occasionnels ; revenus en roubles de décembre 2000. Exemple : en première colonne (pluri-activité), le temps de travail des femmes dans leur activité formelle est de 144 heures par mois, $12 \%$ des femmes déclarent travailler un nombre d'heures nul dans le secteur formel. Leur salaire horaire pour leur activité formelle est de 11,6 roubles par heure et $17 \%$ déclarent avoir un salaire mensuel nul alors qu'elles ont travaillé (lors des 30 derniers jours). Ces femmes ont également une activité informelle qui leur prend 51,8 heures par mois etc. Finalement, le revenu total de leur ménage est de 5580 roubles, $47 \%$ venant des activités formelles et $25 \%$ des activités informelles, le reste des revenus du ménage provenant de transferts qui ne correspondent pas à un emploi ou une activité.

Champ : population en âge de travailler (femmes de 15 à 54 ans et hommes de 15 à 59 ans).

Sources : RLMS, vagues 5-9 in Gignoux J. et Najman B. (2002), "Offre de travail familiale et secteur informel en Russie, 1994-2000 ", mimeo. 
mais il ne peut pas travailler à plein temps dans ce secteur. Cela peut s'expliquer par l'existence de barrières à l'entrée du secteur informel et par les contraintes liées à l'emploi formel.

Deux particularités du marché du travail en Russie apparaissent également : les arriérés de salaires et les congés sans solde. Environ 20 à $25 \%$ des actifs exerçant des emplois formels et 15 à $20 \%$ de ceux exerçant des activités informelles déclarent avoir une activité lors des 30 derniers jours mais leur variable de revenu est nulle (cf. tableau 4). Ceci peut correspondre soit à des arriérés de salaire, soit à la non-déclaration des revenus informels (18). De même, environ $10 \%$ des personnes dans les emplois formels et $3 \%$ dans les activités informelles déclarent avoir un travail formel ou informel mais leur temps de travail est nul. Pour le secteur formel, ce résultat peut s'expliquer par le recours aux congés sans solde à l'initiative de l'entreprise.

\section{Quatre hypothèses à tester}

En Russie, face à la baisse des revenus réels salariés entre 1993 et 1999 (-43,6\%, cf. tableau A de l'annexe 1), les habitants ont développé des stratégies de survie ou parfois d'enrichissement reposant sur la réalisation de revenus supplémentaires. Ces revenus sont déterminés par l'offre de travail, mais cette offre en Russie ne s'exprime pas seulement au travers d'emplois déclarés (formels).

L'impact de ces revenus sur la pauvreté peut être résumé par les hypothèses suivantes.

$\boldsymbol{H 1}$ : Le fait de cumuler deux emplois ou plus informel ou non - réduit la probabilité d'être pauvre (définition monétaire) relativement à un emploi unique (formel ou informel).

$\boldsymbol{H 2}$ : L'activité informelle réduit la probabilité d'être pauvre (définition monétaire) relativement à un emploi formel.

$\boldsymbol{H 3}$ : Le fait de cumuler deux emplois ou plus informel ou non - réduit la probabilité de se sentir pauvre (définition subjective) relativement à un emploi unique (formel ou informel).

H4 : L'activité informelle réduit la probabilité de se sentir pauvre (définition subjective) relativement à un emploi formel.

Les hypothèses $\mathrm{H} 1, \mathrm{H} 2$ et H3 s'expliquent assez facilement car on a mis en évidence (cf. tableau 4) l'importance des rémunérations dans le secteur informel et encore plus nettement dans la pluri-activité. Concernant l'hypothèse H4, une discussion s'impose. En effet, les activités informelles sont par définition de petites activités, relativement instables - et même parfois risquées. Ces activités ne procurent aucune prestation et pas d'avantages sociaux. Une activité informelle n'ouvre pas le droit à la retraite et peut à chaque instant être sanctionnée par l'administration publique (amende de la police, de l'administration fiscale, etc.) (19).

Par ailleurs, l'inefficacité du système de protection sociale (cf. tableau $\mathrm{C}$ en annexe 1), le faible niveau des retraites (en 2000, la retraite représentait environ $30 \%$ du salaire moyen, autour de $30 €$ par mois), la quasi-absence d'indemnisation chômage (environ $4 €$ par mois en 2000) (20) peuvent expliquer que travailler dans le secteur formel ne donne pas nécessairement le sentiment d'être plus riche que le fait de travailler dans l'informel. Ce que peut rapporter un emploi formel a largement perdu de sa valeur en termes monétaires mais aussi en termes de perception de la pauvreté relative. Ces explications sont en partie confirmées par la part décroissante des individus travaillant dans le secteur formel : ils passent de $82 \%$ à $76 \%$ de la population employée entre 1994 et 2000 (cf. tableau 3).

On propose une méthodologie qui permet de tester les hypothèses H1-H4. On travaille d'abord avec le modèle logistique à effets fixes, puis on applique le modèle récursif à équations simultanées (modèle probit bivarié).

\section{La prise en compte de l'hétérogénéité individuelle}

Le premier modèle utilisé pour l'analyse du lien entre pauvreté et participation au marché du travail est un modèle logistique standard avec effets fixes (cf. encadré). La nature des données de panel permet d'analyser la dynamique des

\footnotetext{
18. Ces pourcentages ont été calculés en utilisant les données "brutes ", donc sans l'utilisation de la procédure d'imputation. Pour les calculs des revenus individuels et du ménage, cette procédure d'imputation a été utilisée par les gérants des données RLMS. Il y a des inconvénients liés à cette procédure mais on préfère garder le plus d'observations possibles. Vu la grande quantité des sources de revenus possibles, on perdrait trop d'observations car celles où l'une des sources de revenu manque, sont très nombreuses. Pour la variable de la pauvreté objective, si la personne déclare avoir travaillé pendant les 30 derniers jours, mais ne déclare pas son revenu, la variable est donc imputée. Par ailleurs, le nombre de personnes ayant des arriérés a considérablement diminué après la crise financière de 1998. 19. Les sommes des amendes potentielles pour le travail au noir ne sont pas prises en compte dans les montants déclarés dans l'enquête. Les individus sont supposés déclarer une rémunération reçue de la main à la main.
}

20. Cf. Goskomstat (2001). 
comportements des agents et de prendre en compte leur hétérogénéité inobservée. On considère que la pauvreté a un caractère multidimensionnel et que le nombre de caractéristiques individuelles et de situations non observables - les motivations, l'effort, l'aversion au risque - susceptible de l'influencer justifie l'introduction d'effets fixes. Cette dernière s'explique aussi par les possibles corrélations entre les variables explicatives (l'éducation, la participation au marché du travail, etc.) et les effets individuels non observables. On peut, par exemple, supposer que la motivation (difficilement mesurable) des individus est fortement corrélée avec la participation au marché du travail. On ne peut donc pas, dans ces conditions, utiliser un modèle à effets aléatoires. Le modèle à effets fixes suppose également que, condition- nellement à l'effet individuel, l'influence des variables explicatives observées sur la pauvreté est identique pour tous les individus quelle que soit la période considérée. Enfin, toujours conditionnellement à l'effet individuel, les variables explicatives sont supposées strictement exogènes (21).

Afin d'analyser la pauvreté subjective, un panel non cylindré de 1994 à 2000 a été créé. Il contient 48071 observations représentant 16702 individus (22). On a inclus tous les individus du

21. On ne dispose pas de tests permettant de valider ou non cette hypothèse (Lollivier, 2001).

22. La représentativité du panel pour la Russie dans son ensemble par rapport à l'enquête force de travail du Goskomstat a été vérifiée dans plusieurs travaux récents (par exemple, Najman et Pailhé (2001)).

\section{Encadré}

\section{MODĖLE LOGISTIQUE STANDARD AVEC EFFETS FIXES ET MODĖLE DE PROBIT BIVARIÉ}

\section{Modèle logistique standard avec effets fixes}

Le modèle s'écrit de la manière suivante :

$P_{i t}^{*}=X_{i t} \beta+T_{i t \gamma}+\alpha_{i}+\varepsilon_{i t}$ (équation de pauvreté) [1].

$P_{i t}=1$ si $P_{i t}^{*}>0, P=0$ sinon [2], où :

- $P_{i t}$ est la pauvreté objective ou subjective (deux modalités) ;

- $T_{i t}$ une série de variables indicatives caractérisant le travail ou les statuts sur le marché du travail : avoir un seul emploi salarié (formel), activité informelle ou pluri-activité ;

- $X_{i t}$ est un vecteur des caractéristiques individuelles et du ménage ;

- $\alpha_{i}$ est un effet individuel ;

- $\varepsilon_{i t}$ désigne le terme d'erreur.

En omettant le conditionnement par rapport à $T_{i t} \gamma$ pour simplifier les notations, la spécification logistique implique que :

$P\left(P_{i t}=1 \mid X_{i t}, \alpha_{i}\right)=\exp \left(\alpha_{i}+X_{i t} \beta\right) / 1+\exp \left(\alpha_{i}+X_{i t} \beta\right)$

Chamberlain (1980) a montré que l'estimation simple de ce modèle par le maximum de vraisemblance n'est pas convergente dès lors que $T$ (la durée du panel) est fini. La solution qu'il propose consiste à utiliser la fonction du maximum de vraisemblance conditionnelle. On peut, par exemple, conditionner la fonction du maximum de vraisemblance par le fait que $q$ dates parmi $T$ soient telles que la variable observée soit égale à 1 (Lollivier, 2001). Pour la probabilité décrite dans [3] on obtient la formule (Lollivier, 2001 ; Sevestre, 2002) :

$$
\begin{aligned}
P\left(P_{i t}=1 \mid X_{i t}, \alpha_{i}, \sum_{t=1}^{T} P_{i t}=q\right)= & \exp \left(\sum_{t=1}^{T} P_{i t} X_{i t} \beta\right) / \\
& \sum_{d \in B i} \exp \left(\sum_{t=1}^{T} d_{t} X_{i t} \beta\right)
\end{aligned}
$$

avec : $B_{i}=\left\{d=\left(d_{1}, \ldots d_{T}\right) / d_{t} \in\{0,1\}\right.$ et $\left.\sum_{t=1}^{T} d_{t}=\sum_{t=1}^{T} P_{i t}\right\}$

\section{Modèle de probit bivarié}

Dans le modèle biprobit, les équations représentant la pauvreté s'écrivent :

$P_{i t}^{*}=X_{i t} \beta+T_{i t} \gamma+\varepsilon_{i}$ (équation de pauvreté)

$P_{i t}=1 \mathrm{si} P_{i t}^{*}>0, P_{i t}=0$ sinon

Les équations de participation au marché du travail se formulent ainsi :

$T_{i t}^{\star}=Z_{i t} \theta+\eta_{i t}$ (équation de participation au marché du travail)

$T_{i t}=1 \mathrm{si} T_{i t}^{\star}>0, T_{i t}=0$ sinon [7], où

- $P_{i t}$ est la pauvreté objective ou subjective (deux modalités) ;

- $T_{i t}$ une série de variables indicatives caractérisant le travail ou les statuts sur le marché du travail : avoir un seul emploi salarié (formel), activité informelle ou pluriactivité ;

- $Z_{i t}$ est un vecteur des caractéristiques individuelles et du ménage ;

- $\eta_{i t}$ désigne le terme d'erreur;

avec: $E\left[\varepsilon_{i t}\right]=E\left[\eta_{i t}\right]=0, \operatorname{Var}\left[\varepsilon_{i t}\right]=\operatorname{Var}\left[\eta_{i t}\right]=1$, $\operatorname{Cov}\left[\varepsilon_{i t}, \eta_{i t}\right]=\rho$. 
panel car la pauvreté subjective est définie au niveau individuel. Dans la mesure où la pauvreté objective est définie au niveau du ménage, on désigne un chef de ménage : une femme en âge de travailler (23). L'échantillon contient 18315 observations représentant 6762 individus (ménages).

L'analyse des résultats des régressions logistiques à effets fixes - les estimations sont présentées dans le tableau A de l'annexe 2-, montre que le nombre d'observations a considérablement diminué par rapport à l'échantillon initial. L'estimateur par le maximum de vraisemblance conditionnel utilise uniquement les groupes (individus) pour lesquels la variable expliquée change. Ainsi, les individus restant soit pauvres soit riches pendant la période d'observation sont exclus de l'estimation (24). Pour la pauvreté subjective, par exemple, l'échantillon initial comporte 48071 observations (16 702 individus). Après avoir exclu les valeurs manquantes, le nombre d'observations n'est plus que de 40645 , et correspond à 14436 individus, parmi lesquels 4456 n'ont figuré qu'une seule fois dans le panel, 4356 n'ont jamais été pauvres et 900 ont toujours été pauvres. Finalement, le nombre d'observations « efficaces » est de 18730 représentant 4724 individus (cf. tableau 5).

On peut supposer que les caractéristiques des pauvres transitoires peuvent être différentes de celles des pauvres permanents (25). Pourtant, ces divergences ne devraient pas créer de sérieux problèmes d'interprétation des résultats. Ces deux groupes sont ici assez homogènes, surtout pour la pauvreté objective. Pour la pauvreté subjective, il y a des divergences concernant l'éducation et le lieu d'habitation : les pauvres permanents ont un niveau d'éducation plus faible et habitent plutôt en milieu rural. De plus, la population de pauvres permanents que l'on observe n'est pas nombreuse. Pour la pauvreté objective, par exemple, le nombre total d'individus (ménages) restant toujours pauvres est de 360 parmi les 6762 de l'échantillon initial. Enfin, le panel contient cinq périodes et les personnes restées pauvres pendant quatre ou cinq périodes sont très peu nombreuses $(3 \%$ à $7 \%)$.

\section{Travailler réduit-il la probabilité d'être pauvre?}

Afin de montrer l'importance de l'hétérogénéité individuelle non observée et de mettre en évidence le problème de l'endogénéité, on a également effectué une estimation par un modèle logistique sans effets individuels (cf. tableau A en annexe 2). Il en résulte que, dans le cas du modèle logit à effets fixes, les effets des statuts sur le marché du travail (salarié unique, pluriactivité) sur la pauvreté objective et subjective sont plus élevés et l'effet de l'informel devient significatif. Pour la pauvreté subjective, on ne retrouve pas les mêmes résultats pour l'âge et l'âge au carré. La variable de l'âge au carré devient non significative et le coefficient de la variable âge est de signe négatif. Pour la pauvreté objective, au contraire, les estimations des coefficients de l'âge et de l'âge au carré sont sensiblement plus élevés dans le cas du modèle

\footnotetext{
23. Dans le cas où le ménage comprend deux femmes en âge de travailler, le chef du ménage est la plus âgée. S'il n'y a pas de femmes en âge de travailler dans le ménage, c'est l'homme en âge de travailler qui est pris comme chef du ménage. Si le ménage est composé d'un couple de retraités, la femme est alors le chef du ménage. Les mêmes régressions ont été refaites pour la pauvreté objective avec comme chef du ménage un homme en âge de travailler. On retrouve les mêmes résultats concernant l'impact du marché du travail sur la pauvreté.

24. On a calculé le pourcentage de transitions pour les diverses variables utilisées dans l'estimation par un modèle logistique avec effets fixes. Pour les variables d'intérêt, à savoir les secteurs du marché du travail, l'ampleur des changements est comprise entre $10 \%$ et $35 \%$.

25. Cf. Jenkins (2000), par exemple. Les calculs caractérisant ces deux populations différentes sont disponibles auprès des
} auteurs.

Tableau 5

Caractéristiques de l'échantillon pour le modèle logistique avec des effets fixes sur la pauvreté subjective* $^{*}$

\begin{tabular}{|c|c|c|c|}
\hline & Jamais pauvre & Toujours pauvre & Changement \\
\hline Nombre total d'individus sans valeurs manquantes & \multicolumn{3}{|c|}{14436 individus - 40645 observations } \\
\hline Une seule fois dans le panel & \multicolumn{3}{|c|}{4456 individus (30,87 \% de l'échantillon total) } \\
\hline $\begin{array}{l}\text { Total d'individus } \\
\text { En pourcentage de l'échantillon total (\%) }\end{array}$ & $\begin{array}{l}4356 \\
30,17\end{array}$ & $\begin{array}{l}900 \\
6,23\end{array}$ & $\begin{array}{c}4724 \\
32,72(18730 \\
\text { observations.) }\end{array}$ \\
\hline $\begin{array}{l}\text { En pourcentage de l'échantillon sans ceux qui n'étaient qu'une } \\
\text { fois dans le panel (9 } 980 \text { individus) (\%) }\end{array}$ & 43,65 & 9,02 & 47,33 \\
\hline
\end{tabular}

* Les mêmes calculs pour la pauvreté objective sont disponibles auprès des auteurs.

Source : RLMS, vagues 5 à 9 , calculs des auteurs. 
à effets fixes (forme en U inversé). Différentes explications peuvent être avancées. D'une part les jeunes, dans la période de transition, semblent mieux se débrouiller - accès à des emplois mieux rémunérés - que les adultes d'âge intermédiaire. Une autre explication tient à la présence, en Russie, de travailleurs pauvres durant la période de transition. En effet, en 2000, $64 \%$ des ménages pauvres (en termes monétaires) avaient à leur tête un chef de ménage ayant un emploi. Pour ce qui est des personnes âgées, une solidarité au sein de la famille (entre générations) semble se maintenir.

Les résultats sont aussi instables pour les variables de la structure du ménage. Pour la pauvreté objective, par rapport au couple sans enfant, tous les autres statuts présentent une plus forte probabilité d'être pauvre. La présence des personnes dépendantes, des enfants et/ou des personnes âgées, augmente la probabilité d'être pauvre. Être une personne isolée est aussi défavorable vis-à-vis de la pauvreté objective. Pour la pauvreté subjective, les couples avec enfants se sentent mieux que les couples sans enfants et, au contraire, les célibataires seuls ou avec parents se sentent plus vulnérables. Il s'agit peut-être, dans ce cas, de l'importance d'autres facteurs que les facteurs monétaires: la présence des enfants dans le ménage. Ce résultat sera confirmé ci-après par les régressions à l'aide d'un modèle de probit bivarié : la variable de la taille du ménage est toujours négativement corrélée avec la pauvreté subjective. Les individus se sentent moins pauvres dans les familles nombreuses.

Concernant les données sur l'éducation, l'enquête RLMS contient un grand nombre d'observations incohérentes. Pour près de $20 \%$ des individus, le niveau d'éducation (diplôme obtenu) baisse au cours de la période. En conséquence, on a dû corriger (26) la variable éducation et finalement le nombre de transitions est relativement faible, particulièrement pour l'éducation supérieure (entre $2 \%$ et $3 \%$ ). Les transitions (croissantes) entre niveaux d'éducation plus faibles concernent principalement les jeunes qui n'ont pas encore achevé leur cursus de formation. Ces raisons ont poussé à traiter l'éducation comme une variable constante - et donc absorbée par les effets fixes.

Les résultats de ces deux modèles sont assez différents et ne peuvent pas être qualifiés de stables. Cette instabilité confirme l'hypothèse d'une hétérogénéité significative et met en évidence le problème de l'endogénéité. L'introduc- tion d'effets fixes est d'ailleurs un moyen de réduire l'endogénéité par rapport à un modèle introduisant uniquement des variables observables (27).

Le résultat le plus important du modèle logistique avec des effets fixes est que toutes les variables d'emploi (informel, formel et pluri-activité) sont très fortement corrélées à la pauvreté. Le fait de travailler - quel que soit le statut réduit la probabilité d'être pauvre relativement au fait de se trouver inactif.

\section{Probabilités d'être ou de se sentir pauvre selon la situation sur le marché du travail}

Le problème du modèle précédent est que les variables d'intérêt, à savoir être ou se sentir pauvre, et la participation au marché du travail sont probablement conjointement déterminées. Étant donnée la relation très forte entre la pauvreté et les statuts sur le marché du travail, le modèle récursif à équations simultanées (28) (Greene, 2000) peut être appliqué. Dans ce modèle, les deux équations (la pauvreté et la participation au marché du travail) sont supposées corrélées. Ce modèle est similaire au modèle de probit bivarié simple, à ceci près que la variable dépendante de la deuxième équation apparaît du côté droit de la première équation. Greene (2000) montre que la nature endogène d'une des variables indépendantes de la première équation peut être négligée lors de la formulation du maximum de vraisemblance.

Ce modèle exige pourtant des hypothèses très fortes. Premièrement, on n'exploite plus la double dimension du panel (individuelle-

26. Si un individu donnait un même niveau d'éducation plusieurs années de suite, puis changeait à la baisse ce niveau, on a gardé le niveau d'éducation le plus souvent déclaré. Si l'on ne pouvait observer de répétition on a corrigé la variable éducation en reportant le niveau le plus élevé atteint pour les observations suivantes. Cette correction entraîne certes la surévaluation de l'éducation mais, par ce moyen, on réduit cependant l'erreur de mesure actuelle. On utilise cette variable d'éducation ainsi corrigée dans toutes les régressions (cf. tableaux $A$ à $C$ en annexe 2).

27. Dans la spécification de logit avec effets fixes, le fait de traiter de variable constante la région d'habitation peut paraitre surprenant. Ceci peut s'expliquer par la construction même de la base de données RLMS. Dans la plupart des travaux fondés sur cette base de données (cet article inclus), il est indiqué que les données ont une structure de panel. Pourtant, ce n'est pas un panel "pur " : les individus ayant déménagé depuis les vagues précédentes ne sont pas suivis, à moins de s'installer dans la même localité. II en résulte que les personnes présentes plusieurs fois dans le panel sont celles qui sont restées pendant toute la période de l'enquête à la même adresse. II n'y a pas de mobilité interrégionale. Un tel panel reste vulnérable à un biais de sélection si les raisons du départ sont corrélées avec les variables dépendantes utilisées. Toutefois, c'est la seule source de données disponibles.

28. «Recursive simultaneous equations model ». 
temporelle), laquelle permet de prendre en compte l'hétérogénéité inobservée des agents. Les effets fixes ne peuvent plus être introduits ici. On doit donc supposer qu'ils sont orthogonaux aux variables explicatives. L'hétérogénéité qui existe entre les individus est par ailleurs prise en compte partiellement par l'hypothèse d'une corrélation entre les erreurs des deux équations (29). Deuxièmement, la formule de vraisemblance n'est correcte que s'il y a indépendance entre les termes d'erreur pour les différentes observations, ce qui est difficile à imaginer pour les termes d'erreur relatifs aux diverses périodes pour le même individu (30).

Toutefois, l'intérêt d'un modèle bivarié est de pouvoir évaluer les chances d'observer un individu étant (ou se considérant) pauvre, connaissant sa participation au marché du travail, et de répondre à des questions concrètes : Qui sont ceux qui se sentent (ou qui sont) plus pauvres ? Ont-ils un emploi formel ou bien travaillent-ils dans le secteur informel ?

Le problème est que dans le modèle retenu (cf. les équations [4] et [6] de l'encadré), les variables expliquées doivent être dichotomiques. Dans ce cas, on perd la diversité du marché du travail russe sur laquelle on veut insister. Pour la retrouver, on procède à un découpage qui compare d'abord la probabilité d'être et de sentir pauvre selon le fait d'avoir ou non un emploi. Avoir un emploi quelconque diminue-til la probabilité d'être ou de sentir pauvre ? Ensuite, on analyse les deux groupes suivants à l'intérieur de la population ayant un emploi : les individus pluri-actifs par rapport aux individus ayant une activité unique (formel ou informel) et les salariés (formel) par rapport aux individus ayant des activités informelles (cf. schéma).

Après avoir estimé les coefficients de régression des probits bivariés, on calcule les probabilités conditionnelles d'être ou de se sentir pauvre étant donnée la situation de l'individu sur le marché du travail russe : l'individu a ou non un emploi quel-

\section{Schéma}

\section{Structure du marché du travail russe :} les situations que l'on compare

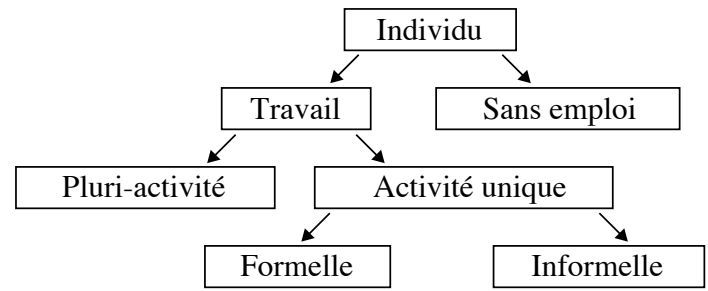

conque, il est pluri-actif ou mono-actif, il travaille dans le secteur formel ou informel.

\section{La précarité liée à l'emploi salarié dans le secteur formel}

Avant de présenter les résultats portant sur les hypothèses concernant les liens entre pauvreté et marché du travail, on va analyser rapidement l'impact des autres variables. L'âge est très significatif à la fois pour la pauvreté subjective et objective. L'introduction de l'âge au carré révèle une relation en forme de $\mathrm{U}$ inversé entre l'âge et le niveau de pauvreté. Pour l'âge, on retrouve les mêmes résultats que pour les régressions avec modèle logistique simple: les personnes jeunes et les personnes âgées sont paradoxalement les moins vulnérables en Russie (cf. supra pour des commentaires sur les formes de solidarité entre générations). Ce résultat est confirmé pour la variable de satisfaction dans d'autres articles (Clark et Maurel, 2001). La variable de sexe masculin n'est pas significative pour la pauvreté objective, et pour la pauvreté subjective, les hommes ont une probabilité plus faible de se sentir pauvres. Il semble que, en Russie (31), les femmes aient une connaissance et une évaluation de leur situation financière plus précises, ou en tout cas plus pessimistes. Les variables d'enseignement technique et supérieur indiquent que l'éducation réduit la probabilité d'être et de se sentir pauvre. Selon les études menées en Russie, le rendement de l'éducation est plus fort pour les enseignements techniques et supérieurs relativement à l'enseignement professionnel (Boeri, 2001).

La taille du ménage réduit la probabilité de se sentir pauvre. Ceci peut s'expliquer par un sentiment de pauvreté plus fort chez les personnes isolées. Ainsi, pour les ménages composés d'une seule personne, $47,8 \%$ se considèrent comme pauvres alors que moins de $30 \%$ des personnes appartenant à des ménages de deux

29. On introduit ainsi des effets aléatoires non individuels mais relatifs à chaque observation.

30. On a effectué les estimations du biprobit en prenant en compte la dépendance des observations à l'intérieur des groupes (les observations relatives aux diverses périodes pour le même individu). En spécifiant l'option " cluster " sous Stata, les obser vations sont supposées être indépendantes entre les groupes, mais pas nécessairement indépendantes à l'intérieur d'un groupe (clusters). Cette spécification affecte les écarts-types estimés et la matrice variance-covariance des estimateurs (VCE), mais pas les coefficients estimés. Les résultats de l'article ne changent pas car les probabilités conditionnelles restent exactement les mêmes. Les résultats sont disponibles auprès des auteurs.

31. En Russie, les hommes semblent être plus heureux que les femmes; on retrouve ce résultat dans les articles qui analysent la satisfaction (Clark et Maurel, 2001 ; Senik, 2002). 
personnes et plus se déclarent pauvres. L'influence de cette variable n'est pas robuste pour la pauvreté objective : elle est négative et significative dans une seule équation simultanée avec l'emploi. Il semble exister en Russie une forte solidarité au sein du ménage qui réduit le sentiment de pauvreté.

Le signe de la variable rurale est toujours positif pour les deux mesures de la pauvreté. Le fait de vivre en milieu rural (par rapport à l'habitat urbain) augmente la probabilité d'être et de se sentir pauvre (32). Le fait de vivre à Moscou ou à Saint-Pétersbourg réduit la probabilité d'être pauvre : les revenus et les possibilités de trouver un emploi y sont considérablement supérieurs par rapport aux autres régions. En revanche, cette variable n'est pas significative pour la pauvreté subjective (33); elle l'est en revanche pour le Caucase (34)

Enfin, l'effet macroéconomique apparaît considérable. Pour les deux mesures de la pauvreté, le signe de la variable vague 8 (égale à un en 1998) est positif : cela traduit l'impact considérable qu'a eu la crise financière de 1998 (35), aussi bien sur les revenus (cf. tableau A en annexe 1) que sur les perceptions de la pauvreté.

Dans toutes les régressions, aussi bien sur la pauvreté subjective que sur la pauvreté objective (à l'exception de la régression sur la pauvreté objective, avec la variable salarié formel et informel en référence), on a retrouvé un coefficient de corrélation $\rho$ significatif. Ceci justifie l'utilisation d'un modèle de probit bivarié qui prend en compte la relation très forte entre la pauvreté et les statuts sur le marché du travail.

On a d'abord comparé la probabilité d'être et de se sentir pauvre pour les personnes qui travaillent et pour celles qui ne travaillent pas (cf. tableau 6). Pour la pauvreté objective, le résultat est prévisible: les actifs ayant un emploi ont une probabilité conditionnelle plus faible d'être pauvre. En revanche, le résultat obtenu sur la pauvreté subjective est plus inattendu. Les individus ayant un emploi ne se sentent pas plus riches et se sentent au contraire plus pauvres que ceux qui n'en ont pas $(0,35$ versus 0,24$)$. Ce résultat peut s'expliquer par la forte hétérogénéité des populations comparées : les individus qui travaillent et ceux qui ne travaillent pas. D'une part, les statuts sur le marché $\mathrm{du}$ travail russe ne sont pas homogènes. Ce résultat traduit aussi la présence, en Russie, de nombreux travailleurs pauvres (en 2000, $44 \%$

32. Dans les données des enquêtes de 1999 sur le budget des ménages du Goskomstat citées par Ovtcharova (2001), 46,2 \% des ménages ruraux sont en dessous du seuil de pauvreté alors que pour les ménages urbains ce chiffre est de 40,9\%. Même durant la période soviétique, les milieux ruraux étaient relativement plus défavorisés que les habitants des grandes villes. Cette discrimination s'est poursuivie durant la transition (Braithwaite, 1994). Elle ne touche pas seulement les revenus mais également l'accès aux services publics, et la qualité de ces services. 33. Les différences entre les niveaux de prix locaux sont prises en compte dans le calcul de la ligne de la pauvreté : les revenus du ménage sont comparés avec les lignes de la pauvreté régionales. 34. Le Caucase nord (Daghestan, Inghouchie) est une région où le secteur informel s'est fortement développé ; c'est probablement la raison pour laquelle elle ressort de manière très significative et négativement dans les régressions.

35. La crise financière que la Russie a connue en août 1998 a représenté un choc considérable. La crise économique s'est traduite par la dévaluation de la devise nationale (de plus de $200 \%$ ), ce qui a abouti à une hausse des prix (de 84,4\%) et à une baisse considérable des revenus de la population (de $27 \%$ entre 1997 et 1999).

\section{Tableau 6}

Résultats des régressions bivariées sur la probabilité d'être et de se sentir pauvre

\begin{tabular}{|c|c|c|}
\hline & $\begin{array}{l}\text { Pauvreté } \\
\text { objective }\end{array}$ & $\begin{array}{l}\text { Pauvreté } \\
\text { subjective }\end{array}$ \\
\hline $\begin{array}{l}\text { Probabilité d'être et de se sentir pauvre si la personne ne travaille pas } \\
\text { Probabilité d'être et de se sentir pauvre si la personne travaille }\end{array}$ & $\begin{array}{c}0,49 \\
(0,22) \\
0,21 \\
(0,15)\end{array}$ & $\begin{array}{c}0,24 \\
(0,14) \\
0,35 \\
(0,17)\end{array}$ \\
\hline $\begin{array}{l}\text { Probabilité d'être et de se sentir pauvre si la personne a une seule activité (formelle ou informelle) } \\
\text { Probabilité d'être et de se sentir pauvre si la personne a plusieurs activités (formel + formel ou occasionnel) }\end{array}$ & $\begin{array}{c}0,31 \\
(0,15) \\
0,10 \\
(0,08)\end{array}$ & $\begin{array}{l}0,29 \\
(0,15) \\
0,03 \\
(0,06)\end{array}$ \\
\hline $\begin{array}{l}\text { Probabilité d'être et de se sentir pauvre si la personne a une seule activité informelle } \\
\text { Probabilité d'être et de se sentir pauvre si la personne a une seule activité formelle }\end{array}$ & $\begin{array}{c}0,18 \\
(0,12) \\
0,31 \\
(0,15)\end{array}$ & $\begin{array}{c}0,09 \\
(0,08) \\
0,28 \\
(0,13)\end{array}$ \\
\hline
\end{tabular}

Lecture : ce tableau présente les probabilités conditionnelles d'être ou de se sentir pauvre étant donnée la situation de la personne sur le marché du travail russe. Elle a ou non un emploi (quel que soit cet emploi), elle est pluri-active ou mono-active, elle travaille dans le secteur formel ou informel. Ces résultats représentent la moyenne des probabilités conditionnelles individuelles. Les écarts-types (variations empiriques des probabilités conditionnelles) sont indiqués en italique et entre parenthèses.

Source : RLMS, vagues 5 à 9 , calculs des auteurs. 
des personnes se déclarant pauvres ont un emploi). D'autre part, les individus qui se déclarent sans emploi peuvent ne pas révéler leurs revenus ou encore posséder d'autres raisons, non monétaires, de se sentir riches.

Pour les personnes ayant plusieurs activités et selon les deux mesures de la pauvreté, la probabilité d'être ou de se sentir pauvre est beaucoup plus faible par rapport à celles qui n'ont qu'une seule activité informelle ou formelle (pauvreté objective: 0,10 versus 0,31 ; pauvreté subjective : 0,03 versus 0,29$)$.

Enfin, le résultat le plus marquant provient de la comparaison des activités informelles avec les emplois formels. Les personnes ayant un emploi formel ont une probabilité d'être et de se sentir pauvre beaucoup plus forte relativement à celles qui ont une activité informelle (pauvreté objective : 0,31 versus 0,18 ; pauvreté subjective : 0,28 versus 0,09$)$. Le secteur informel est souvent présenté comme un secteur instable, où l'activité est incertaine et la volatilité des revenus est importante. On pouvait donc s'attendre au résultat inverse, au moins pour la pauvreté subjective. Ce résultat peut s'expliquer par la baisse significative depuis 1994 des salaires réels dans le secteur formel (cf. annexe 1), mais également par la réduction des avantages liés aux emplois salariés (faible retraite, système de santé en crise, disparition des avantages sociaux, etc.). En effet, l'évaluation subjective de la situation matérielle du ménage intègre pro- bablement un jugement sur la qualité de l'emploi et des avantages auxquels cet emploi donne droit. Ce résultat confirme aussi l'idée que les activités informelles peuvent jouer un rôle important dans la réduction de la pauvreté et dans le développement relativement efficace de revenus alternatifs pour les ménages.

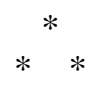

L'emploi formel ne permet donc pas aux actifs de se sentir plus riches, et surtout ne leur permet pas d'être plus riches, par rapport à l'informel. Avoir une activité unique augmente les risques de pauvreté par rapport à la pluri-activité. Travailler dans une activité informelle procure un sentiment de plus grande richesse relative par rapport à travailler dans le secteur formel. Les emplois déclarés ne donnent pas de sécurité ou de stabilité supplémentaire. Ils sont composés de nombreux emplois faiblement rémunérés, le phénomène des travailleurs pauvres semblant se développer dans le secteur formel en Russie.

En Russie, l'emploi formel a ainsi été depuis 1994 le statut le plus défavorable comparé aux autres statuts d'emploi (informel et pluri-activité), et les personnes occupant ce type d'emplois sont devenues vulnérables. Pour échapper à la pauvreté, il vaut mieux actuellement soit cumuler plusieurs emplois (pluri-activité), soit travailler dans le secteur informel.

Les auteurs remercient tout particulièrement Andrew Clark, Jérémie Gignoux, Marina Kolosnitsina, Stéphanie Mahieu, David Margolis, Mathilde Maurel, Claude Montmarquette, Véronique Simonnet, Catherine Sofer, Michel Sollogoub et Antoine Terracol, pour leur lecture et leurs commentaires. Ils remercient également les relecteurs d'Économie et Statistique de leurs avis sur une première version de cet article. Ils remercient enfin les organisateurs de la conférence de Zagreb, en octobre 2002, sur «Les activités informelles dans les pays en transition : dix années d'expériences » qui ont permis de présenter une version préliminaire de cet article.

\section{BIBLIOGRAPHIE}

Banque Mondiale (1996), From Plan to Market, World Development Report.

BERD (1999, 2000, 2001), Transition report, London.

Boeri T. (2001), «Transition with Labour Supply », IZA Discussion Papers, $n^{\circ} 257$.
Braithwaite J. (1994), « From Second Economy to Informal Sector: The Russian Labor Market in Transition », Washington DC., Banque Mondiale, mimeo.

Chamberlain G. (1980), "Analysis of Covariance with Qualitative Data », Review of Economic Studies, $\mathrm{n}^{\circ}$ XLVII, pp. 225-238. 
Clark A. et Maurel M. (2001), « Well-Being and Wage Arrears in Russian Panel Data », Economic Journal of Higher School of Economics, vol. 5, $\mathrm{n}^{\circ} 2$, pp. 179-194.

Gignoux J. et Najman B. (2002), « Offre de travail familiale et secteur informel en Russie, 1994$2000 »$, mimeo.

Goskomstat (2001), Annuaire statistique russe, Russian Statistical Commettee.

Greene W. (2000), Econometric Analysis, Prentice Hall International, Inc.

Grossman G. (1983), « The Second Economy of the USSR » in The Underground Economy in the United States and Abroad, V. Tanzi (ed.). Lexington: Lexington Books.

Jenkins S. (2000), «Modelling Household Income Dynamics », Journal of Population Economics, $\mathrm{n}^{\circ} 13$, pp. 529-567.

Kaliberda S. et Kaufmann D. (1996), «Integrating the Unofficial Economy into the Dynamics of Post-Socialist Economics: Analysis and Evidence », D.T. Banque Mondiale, $n^{\circ} 1691$, Washington.

Kim B.-Y. (2003), « Informal Economy Activities of Soviet Households: Size and Dynamics », Journal of Comparative Economics, $\mathrm{n}^{\circ} 31$, pp. 532551.

Lollivier S. (2001), «Les choix d'activité des femmes en couple : une approche longitudinale », Économie et Statistique, n 349-350, pp. 125-140.

Lollivier S. et Verger D. (1997), «Pauvreté d'existence, monétaire ou subjective sont distinctes », Économie et Statistique, n 308-309310, pp. 113-143.

Najman B. (2000), « Mobilité du travail et secteur informel en Russie et en Ukraine », thèse de doctorat, EHESS, Paris.

Najman B. et Pailhé A. (2001), «Mobilité externe sur le marché de travail russe, 1994-1998. Une approche en termes d'activités », Revue Économique, vol. $52, \mathrm{n}^{\circ} 4$, pp. 861-884.

Ovcharova L. (2001), "What Kind of Poverty Alleviation Policy Does Russia Need? », Russian-
European Centre for Economic Policy Research Paper, Russian Economic Trends, vol. 10, $\mathrm{n}^{\circ} 1$, pp. 7-14, Moscou.

Popkin B., Mozhina M. et Baturin A. (1992), The Development of a Subsistence Income Level in the Russian Federation, Chapel Hill: Carolina Population Center, The University of North Carolina.

Popkin B., Baturin A., Mozhina M. et Mroz T. avec l'assistance de Safronova A., Dmitrichev I., Glinskaya E. et Lokshin M. (1995), The Russian Federation Subsistence Income Level: The Development of Regional Food Baskets and other Methodological Improvements, Chapel Hill: Carolina Population Center, The University of North Carolina.

Russian Economic Trends (2001,2002), différentes parutions, Moscou.

Senik C. (2002), « When Information Dominates Comparaison », document de travail, Delta, Paris.

Sevestre P. (2002), Économétrie des données de panel, Dunod, Paris.

Tchetvernina T., Moscovskaya A., Soboleva I. et Stepantchikova N. (2001), « Labour Market Flexibility and Employment Security. Russian Federation », Employment paper, $n^{\circ} 31$, ILO.

Le lecteur pourra aussi consulter :

Banque Mondiale (1997), Poverty in Russia during transition, ed. by Jeni Klugman.

Gardes F. et Loisy C. (1997), « La Pauvreté selon les ménages : une évaluation subjective et indexée sur le revenu », Économie et Statistique, $n^{\circ} 308$ 309-310, pp. 95-105.

Klugman J. et Braithwaite J. (1998), « Poverty in Russia during the Transition: An Overview », The World Bank Research Observer, vol. 13, $\mathrm{n}^{\circ}$, pp. 37-58.

Lokshin M. et Ravallion M. (2001), «Identifying Welfare Effects from Subjective Questions », Economica, n 68, pp. 335-357.

Ravallion M. et Lokshin M. (2002), « Self-Rated Economic Welfare in Russia », European Economic Review, ${ }^{\circ}$ 46, pp. 1453-1473. 
Tableau A

Évolution de l'emploi, des prix, du PIB, des salaires en termes réels, et niveau du chômage en Russie

En \%

\begin{tabular}{|c|c|c|c|c|c|}
\hline & $\begin{array}{l}\text { Évolution du PIB } \\
\text { en termes réels }\end{array}$ & $\begin{array}{l}\text { Chômage } \\
\text { (1) }\end{array}$ & $\begin{array}{l}\text { Évolution de } \\
\text { l'emploi }\end{array}$ & $\begin{array}{l}\text { Inflation } \\
\text { (2) }\end{array}$ & $\begin{array}{c}\text { Évolution du salaire } \\
\text { réel (3) }\end{array}$ \\
\hline 1991 & $-5,0$ & & $-2,0$ & & n.d. \\
\hline 1992 & $-14,5$ & 5,2 & $-2,3$ & & n.d. \\
\hline 1993 & $-8,5$ & 5,9 & $-1,7$ & 840,0 & 5,4 \\
\hline 1994 & $-12,7$ & 8,1 & $-3,4$ & 204,4 & $-6,5$ \\
\hline 1995 & $-4,1$ & 9,5 & $-3,1$ & 128,6 & $-29,0$ \\
\hline 1996 & $-3,4$ & 9,7 & $-0,6$ & 21,8 & 14,2 \\
\hline 1997 & 0,9 & 11,8 & $-2,0$ & 10,9 & 4,9 \\
\hline 1998 & $-4,9$ & 13,2 & $-2,1$ & 84,5 & $-10,5$ \\
\hline 1999 & 5,4 & 12,6 & 2,8 & 36,8 & $-24,8$ \\
\hline 2000 & 9,0 & 9,8 & 2,2 & 20,1 & \\
\hline 2001 & 5,0 & 8,9 & 0,3 & 18,6 & \\
\hline Variation 1999/1993 & $-26,6$ & & $-11,8$ & & $-43,6$ \\
\hline
\end{tabular}

Sources : BERD, Transition report, 2001, Russian Economic Trends (R.E.T.), 2000.

Tableau B

Les indicateurs officiels caractérisant la pauvreté et les inégalités en Russie

\begin{tabular}{|c|c|c|c|c|c|}
\hline & $\begin{array}{c}\text { Coefficient } \\
\text { de Gini }\end{array}$ & $\begin{array}{c}\text { Coefficient } \\
\text { de la différenciation } \\
\text { des revenus }\end{array}$ & $\begin{array}{c}\text { Les revenus } \\
\text { réels par tête } \\
(1995=100)\end{array}$ & $\begin{array}{c}\text { Ratio du salaire } \\
\text { minimum sur le salaire } \\
\text { moyen (en \%) }\end{array}$ & $\begin{array}{c}\text { \% de la population dont } \\
\text { les revenus monétaires sont } \\
\text { inférieurs au minimum vital }\end{array}$ \\
\hline 1994 & 0,409 & 15,1 & - & 6,6 & 22,4 \\
1995 & 0,381 & 13,5 & 100,0 & 4,3 & 24,7 \\
1996 & 0,387 & 13,0 & 101,3 & 8,0 & 22,0 \\
1997 & 0,401 & 13,5 & 108,2 & 8,8 & 20,7 \\
1998 & 0,399 & 13,8 & 91,4 & 7,9 & 23,3 \\
1999 & 0,400 & 14,0 & 78,5 & 5,5 & 29,9 \\
2000 & 0,399 & 13,8 & 87,3 & 3,8 & 29,1 \\
\hline
\end{tabular}

Lecture : conformément à la méthodologie officielle russe, la ligne de pauvreté est définie par niveau du coût du panier minimal de consommation. Ce panier de consommation comprend la composition minimale des produits de l'alimentation, les denrées non alimentaires et les services nécessaires à la préservation de la santé de la personne et la garantie de son activité vitale. Le minimum vital est quant à lui l'estimation monétaire du coût du panier de consommation, ainsi que les paiements obligatoires et les préparatifs définis au niveau régional. Le minimum vital est calculé en se basant sur la méthodologie établie par le Ministère de l'Emploi de la Russie, le 10 novembre 1992. À partir de 2000, une nouvelle méthodologie est utilisée.

Le coefficient de la différenciation des revenus rapporte la moyenne des revenus des $10 \%$ les plus riches à celle des revenus des $10 \%$ les plus pauvres. Ce coefficient est ainsi dénommé dans la base de données du Goskomstat.

Le montant du salaire minimum en Russie est très faible ce qui explique le niveau du "ratio du salaire minimum sur le salaire moyen ». En effet, le système de protection sociale russe se caractérise par des prestations très peu élevées (cf. tableau C de cette annexe). Le salaire minimum en Russie est loin de couvrir les dépenses minimales d'un travailleur. II sert plutôt comme base pour les différentes amendes et pour d'autres prestations sociales.

Source : Goskomstat, 2001, Russian Economic Trends, décembre 2001. 
Tableau C

La valeur de quelques prestations sociales par rapport au minimum vital

En \%

\begin{tabular}{|c|c|c|c|c|c|c|}
\hline & 1994 & 1995 & 1996 & 1997 & 1998 & 1999 \\
\hline Le salaire minimum & 28 & 10 & 16 & 19 & 18 & 10 \\
\hline La retraite minimale pour la vieillesse & 44 & 27 & 26 & 25 & 29 & 15 \\
\hline $\begin{array}{l}\text { L'allocation sociale : les handicapés depuis l'enfance } \\
\text { - Premier groupe } \\
\text { - Deuxième groupe }\end{array}$ & $\begin{array}{l}73 \\
44\end{array}$ & $\begin{array}{l}54 \\
27\end{array}$ & $\begin{array}{l}52 \\
26\end{array}$ & $\begin{array}{l}50 \\
25\end{array}$ & $\begin{array}{l}57 \\
29\end{array}$ & $\begin{array}{l}30 \\
15\end{array}$ \\
\hline $\begin{array}{l}\text { L'allocation mensuelle pour chaque enfant } \\
\text { - Moins de } 6 \text { ans } \\
\text { - Entre } 6 \text { et } 16 \text { ans (1) }\end{array}$ & $\begin{array}{l}21 \\
18\end{array}$ & $\begin{array}{l}8 \\
7\end{array}$ & - & $\begin{array}{c}- \\
15\end{array}$ & $\begin{array}{c}- \\
14\end{array}$ & - \\
\hline $\begin{array}{l}\text { La bourse minimale pour les étudiants des établissements } \\
\text { d'enseignement supérieur }\end{array}$ & 28 & 10 & 16 & 38 & 36 & 19 \\
\hline $\begin{array}{l}\text { Le minimum vital } \\
\text { - par tête en roubles par mois } \\
\text { - par tête en dollars US (taux de change au } 31 \text { décembre) }\end{array}$ & $\begin{array}{l}86,6 \\
24,4\end{array}$ & $\begin{array}{c}264,1 \\
56,9\end{array}$ & $\begin{array}{c}369,4 \\
66,4\end{array}$ & $\begin{array}{c}411,2 \\
69,0\end{array}$ & $\begin{array}{c}493,3 \\
23,9\end{array}$ & $\begin{array}{r}907,8 \\
33,6\end{array}$ \\
\hline
\end{tabular}

1. À partir de 1996, les allocations pour les enfants de moins de 6 ans ont été regroupées avec celles destinées aux enfants de 6 à 16 ans.

Lecture : il existe deux groupes de handicapés depuis l'enfance définis selon des critères officiels. Le premier groupe regroupe les personnes présentant des handicaps très lourds. Le deuxième groupe concerne les personnes souffrant de dysfonctionnements de l'organisme moins importants. Enfin, le troisième comprend les individus présentant des dysfonctionnements bénins, mais qui provoquent la réduction de la capacité du travail. Les données ne sont pas disponibles pour ce troisième groupe.

Source : Goskomstat (2001). 
Tableau A

Estimation de la pauvreté subjective et de la pauvreté objective sur l'ensemble de l'échantillon

\begin{tabular}{|c|c|c|c|c|}
\hline & \multicolumn{2}{|c|}{ Logistique avec effets fixes } & \multicolumn{2}{|c|}{ Logistique simple } \\
\hline & $\begin{array}{l}\text { Pauvreté } \\
\text { subjective }\end{array}$ & $\begin{array}{l}\text { Pauvreté } \\
\text { objective }\end{array}$ & $\begin{array}{l}\text { Pauvreté } \\
\text { subjective }\end{array}$ & $\begin{array}{l}\text { Pauvreté } \\
\text { objective }\end{array}$ \\
\hline Sexe masculin & - & - & $\begin{array}{c}-0,141^{\star \star \star} \\
(0,024)\end{array}$ & $\begin{array}{c}0,106^{\star} \\
(0,058)\end{array}$ \\
\hline Âge & $\begin{array}{r}-0,036^{*} \\
(0,022)\end{array}$ & $\begin{array}{l}0,251^{\star \star \star} \\
(0,035)\end{array}$ & $\begin{array}{l}0,092^{\star \star \star} \\
(0,004)\end{array}$ & $\begin{array}{l}0,052^{\star \star \star} \\
(0,007)\end{array}$ \\
\hline Âge au carré & $\begin{array}{c}0,028 \\
(0,022)\end{array}$ & $\begin{array}{l}-0,197^{\star \star \star} \\
(0,037)\end{array}$ & $\begin{array}{l}-0,079^{\star \star *} \\
(0,004)\end{array}$ & $\begin{array}{l}-0,081^{\star \star \star} \\
(0,008)\end{array}$ \\
\hline Éducation primaire & - & - & $\begin{array}{l}0,278^{\star \star} \\
(0,034)\end{array}$ & $\begin{array}{l}0,147^{\star \star} \\
(0,058)\end{array}$ \\
\hline Éducation professionnelle & - & - & $\begin{array}{l}0,063 \\
(0,043)\end{array}$ & $\begin{array}{c}0,021 \\
(0,063)\end{array}$ \\
\hline Éducation technique & - & - & $\begin{array}{l}-0,184^{\star \star \star} \\
(0,036)\end{array}$ & $\begin{array}{l}-0,424^{\star \star \star} \\
(0,050)\end{array}$ \\
\hline Éducation supérieure & - & - & $\begin{array}{l}-0,526^{\star \star \star} \\
(0,041)\end{array}$ & $\begin{array}{c}-0,867^{\star \star \star} \\
(0,061)\end{array}$ \\
\hline Avoir un seul emploi salarié & $\begin{array}{l}-0,291^{\star \star \star} \\
(0,064)\end{array}$ & $\begin{array}{l}-0,468^{\star \star \star} \\
(0,098)\end{array}$ & $\begin{array}{l}-0,243^{\star \star \star} \\
(0,031)\end{array}$ & $\begin{array}{l}-0,235^{\star \star \star} \\
(0,047)\end{array}$ \\
\hline Pluri-activité & $\begin{array}{l}-0,573^{\star \star \star} \\
(0,106)\end{array}$ & $\begin{array}{l}-0,908^{\star \star \star} \\
(0,161)\end{array}$ & $\begin{array}{l}-0,352^{\star \star *} \\
(0,062)\end{array}$ & $\begin{array}{l}-0,612^{\star \star \star} \\
(0,095)\end{array}$ \\
\hline Informel & $\begin{array}{c}-0,203^{\star \star} \\
(0,091)\end{array}$ & $\begin{array}{l}-0,474^{\star \star \star} \\
(0,136)\end{array}$ & $\begin{array}{r}-0,083 \\
(0,054)\end{array}$ & $\begin{array}{c}-0,123 \\
(0,082)\end{array}$ \\
\hline Chômeur & $\begin{array}{l}0,351^{* \star *} \\
(0,099)\end{array}$ & $\begin{array}{l}0,435^{\star \star \star} \\
(0,152)\end{array}$ & $\begin{array}{l}0,559^{\star \star \star} \\
(0,061)\end{array}$ & $\begin{array}{l}0,655^{\star \star \star} \\
(0,094)\end{array}$ \\
\hline $\begin{array}{l}\text { Célibataire sans enfant (célibataire seul ou } \\
\text { avec parents) }\end{array}$ & $\begin{array}{l}0,479^{\star \star \star} \\
(0,118)\end{array}$ & $\begin{array}{c}0,059 \\
(0,194)\end{array}$ & $\begin{array}{l}0,500^{\star \star *} \\
(0,039)\end{array}$ & $\begin{array}{l}0,427^{\star \star \star} \\
(0,06)\end{array}$ \\
\hline $\begin{array}{l}\text { Célibataire avec enfants (célibataire seul } \\
\text { avec enfants ou avec enfants et parents) }\end{array}$ & $\begin{array}{c}0,187 \\
(0,116)\end{array}$ & $\begin{array}{l}0,457^{\star *} \\
(0,186)\end{array}$ & $\begin{array}{l}0,443^{\star * *} \\
(0,040)\end{array}$ & $\begin{array}{l}0,675^{\star \star \star} \\
(0,064)\end{array}$ \\
\hline $\begin{array}{l}\text { Couple avec enfants (couple seul avec enfants } \\
\text { ou avec enfants et parents) }\end{array}$ & $\begin{array}{c}-0,189^{\star *} \\
(0,091)\end{array}$ & $\begin{array}{r}0,282^{*} \\
(0,156)\end{array}$ & $\begin{array}{c}0,002 \\
(0,050)\end{array}$ & $\begin{array}{l}0,516^{\star \star *} \\
(0,056)\end{array}$ \\
\hline Constante & - & - & $\begin{array}{l}-3,115^{\star \star} \\
(0,096)\end{array}$ & $\begin{array}{l}-1,478^{\star \star \star} \\
(0,163)\end{array}$ \\
\hline Log-vraisemblance & $-7058,65$ & $-3190,09$ & $-23084,944$ & $-9945,5$ \\
\hline Nombre d'observations & 18730 & 8680 & 40645 & 18315 \\
\hline Nombre de groupes & 4724 & 2220 & - & - \\
\hline
\end{tabular}

Lecture : variable dépendante : pauvreté : $p=1$ si la personne est pauvre (estimation objective et subjective); $p=0$ si la personne est riche. Seuils de significativité : * statistiquement significatif à $10 \% ;{ }^{* *}$ à $5 \%$; ***à $1 \%$.

Variables de référence : inactifs, éducation secondaire, couple sans enfant.

Pour la pauvreté objective on a choisi, comme chef du ménage, une femme en âge de travailler.

Pour l'estimation avec le modèle de logistique simple on a ajouté les variables suivantes : sexe masculin, les variables d'éducation : primaire, professionnelle, technique, supérieure (secondaire en référence), habitat rural, les régions d'habitation : Moscou-St-Pétersbourg, Nord-Ouest, Volga, Caucase, Oural, Sibérie occidentale, Sibérie orientale (Centre en référence), les variables du temps: Vague 5, Vague 6, Vague 8 et Vague 9 (Vague 7 en référence). Les écarts-types sont donnés en italique et entre parenthèses.

Source : données de panel RLMS, vagues 5-9. 
Tableau B

Pauvreté objective (biprobit)

\section{1 - Coefficients et significativité}

\begin{tabular}{|c|c|c|c|c|c|c|}
\hline & $\begin{array}{l}\text { Pauvreté } \\
\text { objective }\end{array}$ & Emploi & $\begin{array}{l}\text { Pauvreté } \\
\text { objective }\end{array}$ & $\begin{array}{l}\text { Pluri- } \\
\text { activité }\end{array}$ & $\begin{array}{l}\text { Pauvreté } \\
\text { objective }\end{array}$ & $\begin{array}{l}\text { Un seul emploi } \\
\text { salarié }\end{array}$ \\
\hline & Coefficient & Coefficient & Coefficient & Coefficient & Coefficient & Coefficient \\
\hline Sexe masculin & 0,006 & $0,391^{\star \star \star}$ & 0,015 & 0,087 & $-0,002$ & $-0,234^{\star \star \star}$ \\
\hline Âge & $0,025^{\star \star \star}$ & & $0,073^{\star * *}$ & $0,032^{\star \star \star}$ & $0,086^{\star \star \star}$ & $0,081^{\star \star \star}$ \\
\hline Âge au carré & $-0,041^{\star \star \star}$ & & $-0,103^{\star \star \star}$ & $-0,046^{\star \star \star}$ & $-0,119^{\star \star \star}$ & $-0,095^{\star \star \star}$ \\
\hline Éducation primaire & $0,238^{\star \star \star}$ & $-0,628^{\star \star \star}$ & $0,214^{\star \star \star}$ & $-0,210^{\star \star \star}$ & $0,214^{\star \star \star}$ & 0,054 \\
\hline Éducation professionnelle & $-0,028$ & $0,129^{\star \star \star}$ & 0,020 & 0,028 & 0,029 & 0,056 \\
\hline Éducation technique & $-0,309^{\star \star \star}$ & $0,247^{\star \star \star}$ & $-0,262^{\star \star \star}$ & $-0,016$ & $-0,246^{\star \star \star}$ & $0,271^{\star \star \star}$ \\
\hline Éducation supérieure & $-0,600^{\star \star *}$ & $0,465^{\star \star \star}$ & $-0,560^{\star \star \star}$ & $0,235^{\star \star \star}$ & $-0,522^{\star \star \star}$ & $0,416^{\star \star \star}$ \\
\hline $\begin{array}{l}\text { Avoir un emploi, pluri-activité, } \\
\text { avoir un seul emploi salarié }\end{array}$ & $0,626^{\star \star \star}$ & & $0,624^{*}$ & & $-0,516^{\star \star}$ & \\
\hline Log de la taille du ménage & $-0,121^{\star \star \star}$ & & 0,048 & & 0,052 & \\
\hline Célibataire seul & & $-0,229^{\star \star \star}$ & & $0,363^{\star \star \star}$ & & $-0,358^{\star \star \star}$ \\
\hline Parent isolé & & $0,782^{\star \star \star}$ & & $0,327^{\star \star \star}$ & & $-0,319^{\star \star \star}$ \\
\hline Couple avec enfants & & $0,873^{\star \star \star}$ & & 0,084 & & $-0,104^{*}$ \\
\hline Célibataire avec parents & & 0,069 & & $0,434^{\star \star \star}$ & & $-0,389^{\star \star *}$ \\
\hline Célibataire avec parents et enfants & & $0,961^{\star \star \star}$ & & 0,170 & & $-0,426^{\star \star \star}$ \\
\hline Couple avec parents & & $0,896^{\star \star \star}$ & & $0,257^{\star}$ & & $-0,165$ \\
\hline Couple avec parents et enfants & & $0,839^{\star \star \star}$ & & $0,176^{*}$ & & $0,190^{*}$ \\
\hline Habitat rural & $0,195^{\star \star \star}$ & $-0,150^{\star \star \star}$ & $0,182^{\star \star *}$ & $-0,162^{\star \star \star}$ & $0,177^{\star \star \star}$ & $0,081^{*}$ \\
\hline Moscou/St-Pétersbourg & $-0,215^{\star \star \star}$ & 0,061 & $-0,250^{\star \star \star}$ & $0,335^{\star \star \star}$ & $-0,276^{\star \star \star}$ & 0,047 \\
\hline Nord-Ouest & $-0,051$ & $0,082^{\star \star}$ & $-0,009$ & 0,083 & 0,024 & 0,002 \\
\hline Volga & $0,231^{\star \star \star}$ & $-0,098^{\star \star \star}$ & $0,263^{\star \star \star}$ & $-0,024$ & $0,290^{\star \star \star}$ & $0,134^{\star *}$ \\
\hline Caucase & $0,237^{\star \star \star}$ & $-0,253^{\star \star \star}$ & $0,234^{\star \star \star}$ & 0,044 & $0,201^{\star \star *}$ & $-0,428^{\star \star *}$ \\
\hline Oural & 0,040 & 0,038 & $0,081^{*}$ & 0,021 & $0,072^{*}$ & $-0,023$ \\
\hline Sibérie Occidentale & $0,173^{\star \star \star}$ & $-0,121^{\star \star \star}$ & $0,098^{\star *}$ & $-0,010$ & 0,076 & $-0,133^{\star *}$ \\
\hline Sibérie Orientale & $0,176^{\star \star \star}$ & $-0,013$ & $0,139^{\star \star \star}$ & $0,278^{\star \star \star}$ & $0,180^{\star \star \star}$ & $-0,170^{\star \star}$ \\
\hline Vague 5 & $-0,661^{\star \star \star}$ & $0,102^{\star \star \star}$ & $-0,585^{\star \star \star}$ & 0,068 & $-0,582^{* \star *}$ & 0,025 \\
\hline Vague 6 & $-0,180^{\star \star \star}$ & $0,060^{*}$ & $-0,123^{\star \star \star}$ & 0,038 & $-0,134^{\star \star \star}$ & $-0,110^{\star}$ \\
\hline Vague 8 & $0,120^{\star \star \star}$ & $-0,048$ & $0,218^{\star \star \star}$ & 0,023 & $0,190^{\star \star \star}$ & $-0,323^{\star \star \star}$ \\
\hline Vague 9 & $-0,193^{\star \star \star}$ & $-0,014$ & $-0,075^{\star}$ & 0,063 & $-0,112^{\star \star}$ & $-0,433^{\star \star *}$ \\
\hline Constante & $-0,841^{\star \star \star}$ & $-0,120^{\star \star \star}$ & $-1,680^{\star \star \star}$ & $-2,194^{\star \star *}$ & $-1,411^{* \star \star}$ & 0,006 \\
\hline Log-vraisemblance & \multicolumn{2}{|c|}{$-19731,21$} & \multicolumn{2}{|c|}{$-9125,73$} & \multicolumn{2}{|c|}{$-8556,75$} \\
\hline Nombre d'observations & \multicolumn{2}{|c|}{18315} & \multicolumn{2}{|c|}{11103} & \multicolumn{2}{|c|}{10215} \\
\hline Corrélation $(\rho)$ & \multicolumn{2}{|c|}{$-0,494$} & \multicolumn{2}{|c|}{$-0,394$} & \multicolumn{2}{|r|}{0,219} \\
\hline$\chi^{2}(\rho)$ & \multicolumn{2}{|c|}{66,78} & \multicolumn{2}{|c|}{5,25} & \multicolumn{2}{|r|}{2,25} \\
\hline Prob. $>\chi^{2}$ (hypothèse $\rho=0$ ) & \multicolumn{2}{|c|}{0,0000} & \multicolumn{2}{|c|}{0,0220} & \multicolumn{2}{|r|}{0,1338} \\
\hline
\end{tabular}

2 - Probabilité d'être pauvre et d'avoir un emploi, probabilité d'être pauvre et pluri-actif et probabilité d'être pauvre et salarié par rapport à l'informel

\begin{tabular}{|l|c|}
\hline & $\begin{array}{c}\text { Probabilités } \\
\text { conditionnelles }\end{array}$ \\
\hline Probabilité d'être pauvre si la personne ne travaille pas & $0,49(0,22)$ \\
Probabilité d'être pauvre si la personne travaille & $0,21(0,15)$ \\
\hline Probabilité d'être pauvre si la personne a une seule activité (formelle ou informelle) & $0,31(0,15)$ \\
Probabilité d'être pauvre si la personne a plusieurs activités (formel + formel ou occasionnel) & $0,10(0,08)$ \\
\hline Probabilité d'être pauvre si la personne a une seule activité informelle & $0,18(0,12)$ \\
Probabilité d'être pauvre si la personne a une seule activité formelle & $0,31(0,15)$ \\
\hline
\end{tabular}

Lecture : variable dépendante : pauvreté : $p=1$ si la personne est pauvre (estimation objective) ; $p=0$ si la personne n'est pas pauvre. Emploi : $t=1$ si la personne a un emploi ; $t=0$ si la personne $n$ 'a pas d'emploi. Seuils de significativité : * statistiquement significatif à $10 \%$; ** à $5 \%$; ** à $1 \%$. Les écarts-types (variations empiriques des probabilités conditionnelles) sont indiqués entre parenthèses et en italique.

Variables de référence : éducation secondaire, couple sans enfant, habitat urbain, région Centre, vague 7.

Source: données de panel RLMS, vagues 5-9. 
Tableau C

Pauvreté subjective (biprobit)

\section{1 - Coefficients et significativité}

\begin{tabular}{|c|c|c|c|c|c|c|}
\hline & $\begin{array}{l}\text { Pauvreté } \\
\text { subjective }\end{array}$ & Emploi & $\begin{array}{l}\text { Pauvreté } \\
\text { subjective }\end{array}$ & $\begin{array}{l}\text { Pluri- } \\
\text { activité }\end{array}$ & $\begin{array}{l}\text { Pauvreté } \\
\text { subjective }\end{array}$ & $\begin{array}{l}\text { Un seul emploi } \\
\text { salarié }\end{array}$ \\
\hline & Coefficient & Coefficient & Coefficient & Coefficient & Coefficient & Coefficient \\
\hline Sexe masculin & $-0,074^{\star \star \star}$ & $0,334^{\star \star \star}$ & $-0,217^{\star \star \star}$ & $0,265^{\star \star \star}$ & $-0,191^{\star \star \star}$ & $-0,156^{\star \star}$ \\
\hline Âge & $0,045^{\star \star \star}$ & & $0,045^{\star \star \star}$ & $0,054^{\star \star \star}$ & $0,064^{\star \star \star}$ & $0,068^{\star \star \star}$ \\
\hline Âge au carré & $-0,039^{\star \star \star}$ & & $-0,041^{\star \star \star}$ & $-0,077^{\star \star \star}$ & $-0,063^{\star \star \star}$ & $-0,077^{\star \star \star}$ \\
\hline Éducation primaire & $0,072^{\star \star \star}$ & $-0,703^{\star \star \star}$ & $0,221^{\star \star \star}$ & $-0,125^{\star \star \star}$ & $0,203^{\star \star \star}$ & 0,026 \\
\hline Éducation professionnelle & $0,073^{\star \star \star}$ & $0,311^{\star \star \star}$ & 0,019 & $0,084^{\star *}$ & 0,029 & $0,084^{\star \star}$ \\
\hline Éducation technique & $-0,088^{\star \star \star}$ & $0,303^{\star \star \star}$ & $-0,157^{\star \star \star}$ & $0,092^{\star \star \star}$ & $-0,117^{\star \star \star}$ & $0,242^{\star \star \star}$ \\
\hline Éducation supérieure & $-0,269^{\star \star \star}$ & $0,451^{\star \star \star}$ & $-0,352^{\star \star \star}$ & $0,268^{\star \star \star}$ & $-0,289^{\star \star \star}$ & $0,392^{\star \star \star}$ \\
\hline $\begin{array}{l}\text { Avoir un emploi, pluri-activité, } \\
\text { avoir un seul emploi salarié }\end{array}$ & $-0,539^{\star \star \star}$ & & $1,186^{\star \star \star}$ & & $-0,888^{\star \star \star}$ & \\
\hline Log de la taille du ménage & $-0,100^{\star \star \star}$ & & $-0,112^{\star \star \star}$ & & $-0,101^{\star \star \star}$ & \\
\hline Célibataire seul & & $-0,229^{\star \star \star}$ & & $0,200^{\star \star \star}$ & & $-0,429^{\star \star \star}$ \\
\hline Parent isolé & & 0,013 & & $0,135^{\star \star \star}$ & & $-0,296^{\star \star *}$ \\
\hline Couple avec enfants & & $0,775^{\star \star \star}$ & & $-0,020$ & & 0,000 \\
\hline Célibataire avec parents & & $-0,399^{\star \star \star}$ & & $0,266^{\star \star \star}$ & & $-0,306^{\star \star \star}$ \\
\hline Célibataire avec parents et enfants & & $0,282^{\star \star \star}$ & & 0,022 & & $-0,318^{\star \star \star}$ \\
\hline Couple avec parents & & $0,266^{\star \star \star}$ & & 0,028 & & 0,037 \\
\hline Couple avec parents et enfants & & $0,811^{\star \star \star}$ & & 0,012 & & 0,061 \\
\hline Habitat rural & $0,064^{\star * \star}$ & $-0,094^{* \star *}$ & $0,193^{\star \star *}$ & $-0,184^{\star \star \star}$ & $0,173^{\star \star \star}$ & $0,067^{\star \star}$ \\
\hline Moscou/St-Pétersbourg & 0,008 & $0,091^{\star \star \star}$ & $-0,023$ & $0,242^{\star \star \star}$ & 0,016 & $-0,107^{\star \star}$ \\
\hline Nord-Ouest & 0,011 & $0,129^{\star \star \star}$ & 0,038 & $0,081^{*}$ & $0,092^{* \star}$ & $0,085^{*}$ \\
\hline Volga & $0,070^{\star \star \star}$ & $-0,098^{\star \star \star}$ & $0,194^{\star \star \star}$ & $-0,040$ & $0,208^{\star \star \star}$ & 0,055 \\
\hline Caucase & $-0,249^{\star \star \star}$ & $-0,222^{\star \star \star}$ & $-0,182^{\star \star \star}$ & 0,034 & $-0,229^{\star \star \star}$ & $-0,408^{\star \star \star}$ \\
\hline Oural & $-0,051^{\star *}$ & 0,028 & $-0,020$ & $-0,061$ & $-0,021$ & 0,019 \\
\hline Sibérie Occidentale & $-0,048^{\star}$ & $-0,064^{\star \star}$ & $-0,035$ & $-0,013$ & $-0,027$ & $-0,001$ \\
\hline Sibérie Orientale & $-0,017$ & $0,061^{\star \star}$ & $-0,043$ & $0,180^{\star \star \star}$ & $-0,009$ & $-0,076^{\star}$ \\
\hline Vague 5 & $-0,053^{\star \star}$ & $0,113^{\star \star \star}$ & $-0,042$ & $0,094^{* \star}$ & 0,001 & $0,114^{\star \star *}$ \\
\hline Vague 6 & 0,022 & $0,074^{\star \star \star}$ & $0,062^{\star \star}$ & 0,006 & $0,062^{\star \star}$ & $-0,020$ \\
\hline Vague 8 & $0,144^{\star \star \star}$ & $-0,081^{\star \star \star}$ & $0,171^{\star \star \star}$ & $-0,010$ & $0,137^{\star \star \star}$ & $-0,219^{\star \star \star}$ \\
\hline Vague 9 & $-0,180^{\star \star \star}$ & $-0,030$ & $-0,163^{\star \star \star}$ & 0,020 & $-0,220^{\star \star \star}$ & $-0,336^{\star \star \star}$ \\
\hline Constante & $-1,132^{\star \star \star}$ & $-0,165^{\star \star \star}$ & $-1,576^{\star \star \star}$ & $-2,438^{\star \star \star}$ & $-1,101^{\star \star \star}$ & 0,056 \\
\hline Log-vraisemblance & \multicolumn{2}{|c|}{$-45219,8$} & \multicolumn{2}{|c|}{$-19033,019$} & \multicolumn{2}{|c|}{$-17953,347$} \\
\hline Nombre d'observations & \multicolumn{2}{|c|}{40645} & \multicolumn{2}{|c|}{23016} & \multicolumn{2}{|c|}{20932} \\
\hline Corrélation $(\rho)$ & \multicolumn{2}{|c|}{0,206} & \multicolumn{2}{|c|}{$-0,594$} & \multicolumn{2}{|r|}{0,397} \\
\hline$\chi^{2}(\rho)$ & \multicolumn{2}{|c|}{30,16} & \multicolumn{2}{|c|}{12,44} & \multicolumn{2}{|c|}{15,92} \\
\hline Prob. $>\chi^{2}$ (hypothèse $\rho=0$ ) & \multicolumn{2}{|c|}{0,0000} & \multicolumn{2}{|c|}{0,0004} & \multicolumn{2}{|r|}{0,0000} \\
\hline
\end{tabular}

2 - Probabilité de se sentir pauvre et d'avoir un emploi, probabilité de se sentir pauvre et d'être pluri-actif et probabilité de se sentir pauvre et d'être salarié par rapport à l'informel

\begin{tabular}{|l|c|}
\hline & Probabilités conditionnelles \\
\hline Probabilité de se sentir pauvre si la personne ne travaille pas & $0,24(0,14)$ \\
Probabilité de se sentir pauvre si la personne travaille & $0,35(0,17)$ \\
\hline Probabilité de se sentir pauvre si la personne a une seule activité (formelle ou informelle) & $0,29(0,15)$ \\
Probabilité de se sentir pauvre si la personne a plusieurs activités (formel + formel ou occasionnel) & $0,03(0,06)$ \\
\hline Probabilité de se sentir pauvre si la personne a une seule activité informelle & $0,09(0,08)$ \\
Probabilité de se sentir pauvre si la personne a une seule activité formelle & $0,28(0,13)$ \\
\hline
\end{tabular}

Lecture : variable dépendante : pauvreté : $p=1$ si la personne est pauvre (estimation objective); $p=0$ si la personne n'est pas pauvre. Emploi : $t=1$ si la personne a un emploi ; $t=0$ si la personne $n$ 'a pas d'emploi. Seuils de significativité : * statistiquement significatif à $10 \%$; ** à $5 \%$; ** à $1 \%$. Les écarts-types (variations empiriques des probabilités conditionnelles) sont indiqués entre parenthèses et en italique.

Variables de référence: éducation secondaire, couple sans enfant, habitat urbain, région Centre, vague 7.

Source: données de panel RLMS, vagues 5-9. 